\title{
Security Issues in Web Services: A Evaluation and Advancement Perspective Concerning Research Agenda
}

\author{
Sunny Kumar \\ Asst Proff \\ SSM College,Dinanagar
}

\author{
Shamsher Singh, PhD \\ Asst Proff \\ SRPAB College \\ Pathankot
}

\author{
Amandeep Singh \\ Research Scholar \\ Guru Kashi University, \\ Talwandi Sabbi,Bathinda
}

\begin{abstract}
Web Service is comparatively emerging and also a significant area. The security challenges concerning Web Services in a dispersed environment are really a significant concern of research. Web service security measures are amongst the steady thrusts aspects of research both in industry as well as in academia. The existing efforts are primarily concentrating on specialized security issues of Web Services. The research is carried out according to the general framework of security issues. It is worthwhile for web service security to completely focus upon subject areas including access control, authentication, consistency in information, and also level of privacy. Security is so essential simply because web services communicate on a program to program factor. Main objective with these recommended efforts would be to explain the approach as well as applications of Multi-Part MultiSignature Document in the work flow environment as well as compares anywhere between XML signature security difficulties with the MPMSD. Furthermore, this work highlights the long term issues with regard to research as well as experimentation underneath the perspective of MPMSD
\end{abstract}

\section{Keywords}

Security, SOC, Web Services, SOA, SOAP, XML, WSDL, UDDI, Single-Sign-On, Multi-Part MultiSignature Document, Digital Signature, XML Signature, DoS.

\section{INTRODUCTION}

Web services have expanded to become more popular with application developers and the technology represents an important way for businesses to communicate with each other and with clients. Web services do not provide the user with a GUI (Graphic User Interface), but they share business logic, data and processes through a programmatic interface across a network ${ }^{[1]}$

\subsection{Service Oriented Computing}

Service Oriented Computing (SOC) ${ }^{[7]}$ is a most recent inflection regarding computer science that contemplate a trend in direction of platform and also language autonomy regarding the components. It is comparatively an innovative new as well as appropriate area. It possesses a great approach to formulate

a new distributed application. SOC assists with implementing along with configuring distributed software systems in a approach that delivers work productivity as well as excellence alongside service-orientation. Services are merely a mean for the building distributed applications, which in turn focusing primarily about how these programs are designed and exactly how services should operate collectively. SOC comes with three positive indigenous capabilities: (i) description, (ii) discovery and (iii) communications. Designers put into practice SOC native possibilities using Web Service Description Language (WSDL) for explanation, Universal
Description, Discovery Integration (UDDI) for discovery and Simple Object Access Protocol (SOAP) for communications. Web service is the present technology of SOC. Security is definitely a significant issue of services for the serviceoriented applications the template, and replace the content with your own material

\subsection{Service Oriented Architecture}

A Service Oriented Architecture (SOA) is primarily an accumulation of services. These types of services converse with one another. SOA describes an fundamental interaction in between software professionals as an exchange of messages in between service requesters as well as service operators. Clientele tend to be software agents that petition the writ of execution of a service. Providers are software agents that offer the service. The fundamental SOA is an association of three features: a service provider, a service requester (buyer) and also a service registry. The actual fundamental interaction involve distribute, discover as well as constipate procedures. The service requester works on the find operation in order to get access to the service description originating from a discovery agency, as well as utilizes the service description to bind along with the vendor as well as conjure the service or perhaps communicate with service execution.

\subsection{Web Service}

Web Services ${ }^{[9]}$ are slackly conjugate self-contained, selfdescribing and also standard programs which really can be characterized, published, established as well as invoked over a network. Web service can be offered upon any sort of platform and may also be developed in any programming language. Web Services primarily incorporate the three duties of SOA: service provider, service requester as well as service agent. A service provider could possibly be a marketplace, small business or perhaps an organization perfect for delivering service. A requester also can be quite a company or maybe a business that is in need of assistance of the service, whereas the agent is a place, organization or perhaps a mechanism which enables you to both service provider and service requester to go through each other.

\section{WEB SERVICE SYSTEM}

The technological innovation that particular form the substructures of Web Services are generally SOAP, WSDL, and UDDI.

\subsection{SOAP}

SOAP is primarily a stateless, one-way communication interchange inflection that enables applications to develop more professional relationship patterns (e.g., request/response, request/multiple reactions, etc.) by incorporating one-way exchanges alongside qualities offered by an fundamental protocol and/or application-specific facts. SOAP does not by itself determine any kind of application semantics ${ }^{[11]}$ for instance a programming version or perhaps effectuation 
focused semantics, for instance, distributed trash set. It preferably characterizes an easy process for illustrating application semantics by offering a conventional product packaging version as well as scribing mechanisms for encryption important information within modules.

Even Though SOAP provides an outstanding framework for important information interchange, it is lacking in semantics pertaining to the application-specific information it delivers, such as the routing of SOAP information, reliable information exchange, firewall traversal, etc. Additionally, SOAP comes with a comprehensive classification of the essential actions considered by a SOAP node on receiving a SOAP message [12]

At its core, a SOAP information incorporates a extremely straight forward framework: an XML element alongside two children elements, one which contains their header and the another the body. The header items as well as the entire body elements are also illustrated in XML.SOAP messages can be transmitted over HTTP for the runtime implementation. The HTTP communications protocol perform the linking feature for the primary fundamental interaction somewhere between computer networks

The Soap Header part could have a set of child elements that describe message operating that the transmitter anticipates a recipient to accomplish.

$<$ Soap: Envelope $\longrightarrow$

$<$ Soap: Header (optional)>

$<$ Soap: Body > (mandatory)

$<$ get Quote symbol = “__ $" />$

</Soap: Body>

</Soap: Envelope>

Example 1: A Simple SOAP message.

- SOAP envelope is employed for encapsulate the SOAP message.

- SOAP header is the elective portion of the SOAP protocol. Header consists of information towards SOAP node, the processor of the SOAP message, how to process the SOAP message. This might be verification, routing etc.

- Soap body consists of the targeted towards SOAP message receiver.

- Get Quote component is the child of SOAP body.

\subsection{WSDL}

Web Service Description Language(WSDL) is used to illustrate the features of the services.

As soon as the requester receives the WSDL document for all the campaigner Web service, it needs to be validated. The most convenient technique of doing this particular is to provide a digital key signature of the WSDL document for the requester to use. Requesters cannot connect to most service providers without having some sort of authentication. WSDL v1.1 doesn't necessarily provide internal mechanism for signing WSDL documents. WSDL v1.1 really doesn't provide a technique for specifying the security specifications of a Web service. Foreseeable future versions of WSDL are scheduled to have this particular feature.

\subsection{UDDI}

Universal Description Discovery and Integration (UDDI) can be used as a registry of information for the Web Services. This simply means to publish and discover important information. UDDI service is an industry-wide undertaking to carry perhaps the most common traditional for business tobusiness (B2B) integration. It specifies a group of ordinary interfaces for accessing a data source of Web services. The objective of UDDI is to allow for users to discover obtainable Web services as well as communicate with them dynamically. The procedure can easily be split up into three phases: Browsing (discovery), Binding and executing (soapceient website).

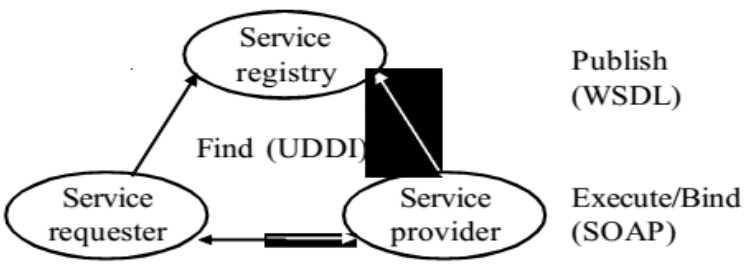

Fig 1 Web Service Model

Web Service is an appealing as well as efficient innovation for the advancement of distributed application as well as for consolidation. But for wide acceptability by the programmers as well as prospective buyers in business-to-business(B2B) and business-to-consumers(B2C) circumstances, it should be secured. Subsequently study of security problems in Web Services is an accomplished demand for the time.

\section{IMPORTANT SECURITY FRAMEWORK}

Security in every system tends to be examined underneath a prevalent general framework. Web Service is certainly not an exception. The framework is comprised of the subsequent issues.

\subsection{Authentication}

Authentication is apprehensive because of the establishment of the evidence of identifications of entities within a system. The entity might be a user, a process or perhaps a service. Masquerading is a conventional attack in authentication approach.

\subsection{Authorization}

As soon as an entity has been authenticated, the subsequent issue is to determine which operations the entity is permitted to do and on what resources. The authorization mechanism works with approving as well as ratifying privileges of authenticated entities.

\subsection{Secrecy}

The matter associated with secrecy determine that only the sender as well as the supposed recipient ought to be in a position to gain access to the information in the message. An unauthorized individual really should not be allowed to access a message. It is attained by encryption as well as decryption of messages. An encryption algorithm can be used to transform plaintext into cipher text. Generally there are a couple of types of encryption as a general rule use: symmetric and asymmetric encryption. In symmetric encryption, the decryption key is the exact same as the encryption key as well as in asymmetric encryption; the decryption key is absolutely not identical to the encryption key. Eavesdropping is an accomplished classique attack in secrecy. 


\subsection{Non-Repudiation}

There are certainly circumstances whenever a individual transmits a message, and later on disowns it. Refusal could possibly be upon sending, receiving or perhaps on the moment of sending or obtaining the message likewise.

\subsection{Accessibility}

The challenge of accessibility claims that resources, services needs to be accessible to certified parties all of the time. Denial of Service (DoS) is usually a classique attack on availability.

\subsection{Integrity}

Whenever information in a message is altered during the course of transmissions, then the integrity regarding the message is destroyed. Data reliability is dependent on mathematical algorithmic regulation acknowledged hashing algorithms. A hashing algorithm requires a block of information as input as well as generates a reduced piece of data as output. This particular output is occasionally known as a digest of the information. If the information is definitely a message, it is known as a message digest. MD5 as well as SHA are the conventional hashing algorithms.

\section{EXCLUSIVE SECURITY}

\section{CHALLENGES FOR WEB SERVICES}

Beneath the basic framework reviewed in upcoming section , specific security issues associated with Web services tend to be talked about as follows:

\subsection{Verification for Web Services}

In SOA, the three functions: requester, service provider and registry need to be authenticated throughout composition, binding and execution of Web Services. Whenever a new service is comprised using established services, the explanations of which have been provided by the registry, then the registry is usually to be authenticated by the requester. Moreover, if it is not really public service, the requester is likely to be required to be peer authenticated by the registry. Likewise during execution of services also, the requester as well as the service provider may prefer to peerauthenticate with one another. Web Services may perhaps be susceptible to man-in-the attack, masquerading attack during the course of composition, constricting as well as execution. An additional appropriate concern is simple tips to figure out that the explanation of small amount supplied by the registry as well as the genuine service offered by the provider for binding are identical. Some form of official certification with this relationship in between explanation as well as the genuine service is required. Can the registry function also employ certifying authority in this situation? If so, it could be a trustworthy Third Party (TTP). However whether off-line, online or perhaps in-line TTP demands even more investigation.

\subsection{Verification Strategies}

\subsubsection{Single-sign-on}

as soon as the end-user has been authenticated through its characteristics login-id and security password, it should be authenticated once again for communicating along with the other services. Once the end-user signs onto an internet site right after which a SOAP request tend to be prepared on the user's behalf, the route amongst several Web Services is founded having user's login-id as well as password. This particular functionality is referred to as Single-sign-on.

Federated-trust: the moment the end-user has been authenticated using its capabilities login-id and password, the route amongst multiple Web Services is likely to be established based on their trust relationships. This particular mechanism is referred to as Federated-trust.

WS-Trust: To route amongst numerous Web Services and the entrust relationship needs to be established among a variety of services. The trust relationship amongst multiple services tends to be either direct or brokered

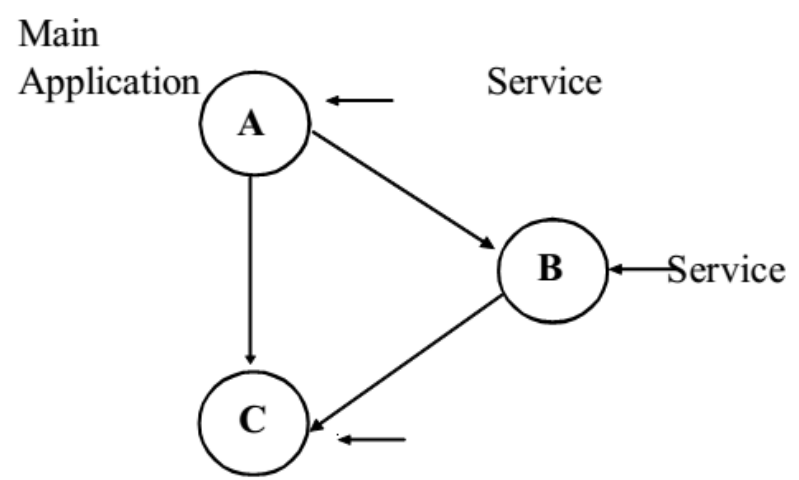

Fig 2.Figure of Multihopping

Let us assume,service A authenticates service B $\Rightarrow$ service A relies on service $B$, service $B$ authenticates service $C \Rightarrow$ service $B$ relies on service C. Therefore, service A authenticates service $\mathrm{C} \Rightarrow$ service $\mathrm{A}$ relies on service $\mathrm{C}$. The preceding illustration demonstrates the situation of Multihopping, meaning that the path through a number of Web Services. In addition, it describes the concept of WSrouting, where SOAP information is to route through a number of Web Services. The idea of WS-routing is certainly not same as with Single-sign-on and Federated-trust, because the former considers merely the SOAP messages has to route amongst a number of Web Services whereas the later originate from authentication and reliance establishment among multiple Web Services. So far security obstacles are preoccupied, the security mechanisms like Single-sign-on and Federated-trust may be configured at the top of WS-routing.

\subsection{Affirmation for Web Services}

An authorization conclusion is necessary for the destination Web Services for getting important information regarding end-user who signs as soon as for routing the SOAP request among multiple Web Services. Whenever multiple web services are now being operate in promptly succession and also time period is actually of the essence, it is actually essential that the expense of endorsement not really occur on every occasion when an additional web service is now being run. SAML (Security Assertion Markup Language) and XACML (eXtensible Access Control Markup Language) are the two technologies that are used to determine authentication along with authorization information. The accessibility control mechanism deals alongside two strategies specifically, Role Based Access Control (RBAC) and Context Based Access Control (CBAC).

In the entire world of information security, the definition of confidentiality can be used to mention to the necessity for information in transit anywhere between two communicating parties not to ever be accessible to the third parties that could try to interrupt within the communications. XML encoding could possibly be the technology utilized for confidentiality in Web service security system. It acts as the premise for other security technologies including XML signature. Basic evidence of origin security specifications of Web services is likely to be tackled by XML signature available nowadays. 
However for specific security specifications in work flow environment, such as multi-signature and multi-part multisignature the current variation of XML signature might have restrictions. Analyst investigating in this direction.

\subsection{For Non-Repudiation Web Services}

Digital signature is certainly not adequate for non-repudiation protocol. Sensible non-repudiation protocol as well as its alternatives need a fundamental arbiter as being a TTP. This might be a obstruct for the future secure of Web services. For the general public Web services non-repudiation may possibly not be a significant issue. But the conventional Web services of e-Governance and professional services in opposition to payment, non-repudiation is regarded as the primary issues to be resolved. XML signature is the technology which can be additionally useful for non-repudiation protocol.

\subsection{Accessibility for Web Services}

Accessibility is definitely a significant problem within Web service security. Certainly one of the opportunities of refusing availability is because of the DoS attack. A DoS attack is designed to use up all of the information of a service which makes it unavailable to prospective users.

\subsection{Trustworthiness for Web Services}

Integrity of Web service is especially associated with the WSDL file, which identifies the benefits of a Web service. Whenever a service requester conveys with the registry for an appropriate service, WSDL file is the foundation of choosing the service during composition. But when it is diluted during transit or storage it might probably incorporate falsehoods, which is actually a result in denial of the service throughout composition and breakdown too as during the course of execution of the service. XML signature will be the technology which you can use for information integrity.

\section{CONDEMNATION ON PRESENT TECHNOLOGIES}

\subsection{WS-Security}

WS-Security is a foundation that supposed to have been used in combination with various other Web Services as well as application specific standards to support numerous types of security models. WS-Security [5] doesn't necessarily claim that they can provide an entire alternative in order to protecting Web services. The XML signature as well as XML encoding descriptions incorporate classique strategies for digitally signing and also encrypting XML documents including SOAP messages. Definitely not exclusively can completely documents be signed or perhaps encoded, but additionally specific parts. WS-Security describes exactly how XML signature data tends to be incorporated into a SOAP message. This provides persistent confidentiality beyond a single SOAP communication.

\subsection{Secure Socket Layer}

(SSL) is a protocol or perhaps technology, which can be used to shield organizations from web Service Security problems. SSL used in encoding approach, which are in turn utilized to make usage or data protection. SSL produces a protected passageway somewhere between originator as well as desired destination computers dependent on public key encoding approach. A frequent appropriate measure is to transmit messages over a reliable connection this is certainly using SSL. For example, an SSL connection in between two points could possibly be adequate for convenient applications. For multiple Web Services, complete information or perhaps individual part of messages is likely to be encoded as well as signed to preserve the privacy as well as integrity of Web Service messages [5].

\subsection{XML Encryption}

XML Encoding offers end-to-end security for applications that necessitate secure change of structured data. XML encoding is primarily ensuring secrecy to encrypt the XML data. XML formulated encoding is the natural way to handle requirements for safety measures in data interchange applications. XML Encoding is certainly not designed to substitute or perhaps supersede Secure Socket Layer (SSL). Rather, it possesses a mechanism for security specifications that aren't protected by SSL. XML encoding is fantastic confidentiality. XML Encryption doesn't expose any other emerging cryptography algorithms or perhaps strategies. RSA Encryption might still be applied for the authentic encoding.

\subsection{SAML}

Security Assertion Markup Language is a protocol for declarative verification as well as endorsement information. Moreover it provides qualities of an end-user in XML format. It permits important information to be put on a SOAP message. SAML servers tends to be accessed for the verification as well as authorization data in order to really permit Single Sign-On (SSO). If the receiver of this particular SOAP message relies on the transmitter of the SAML data, the end user can certainly be legitimate for the Web Service.

\subsection{XACML}

eXtensible Access Control Markup Language or XML-Access Control Markup Language (XACML) is intended to express accessibility control guidelines in XML format. Even though the two technologies usually are not expressly connected, XACML may be utilized in combination with SAML. An authorization decision indicated within a SAML statement might have been dependent on guidelines portrayed in XACML.

\section{CONTRIBUTION}

The primary purpose of this particular insubstantial is usually to do the evaluation work on ordinary security problems as well as exclusive security concerns of WSS. From our personal research it is located that service authentication tends to be enclosed as being a brand new item of specialized security issue in security mechanisms like WS-Routing as well as Multi-hopping. Additionally we have emphasized some latest security measures issues within this paper. We've reviewed on security mechanisms such as federated trust, single-sign-on as well as WS-trust. The rest of the parts of this particular paper tend to be systematic by the controversy on current technologies along with a possible solution of the issue has provided on talking about various other technologies such as digital signature, XML signature and MPMSD. Ultimately, we conclude our conversation with some upcoming directions.

\section{ENDORSED SOLUTION}

The primary intention associated with the paper would be to bring into focus the evaluation of security issues in Web services and appropriately we have discussed a frequent framework of general security concerns. Additionally we have discussed some new security concerns as part of Web Service Security(WSS) along with their attacks. Listed below are some technologies which can provide an optimum solution of security mechanisms.

Digital Signature[2] : it is actually liberated from the signer's identify as well as handwritten signature 
Digital Signature:- $\{\{\delta \mathrm{m}\} \mathrm{sk}(\mathrm{A}) \mathrm{m}, \mathrm{A}\}$

\begin{tabular}{|c|c|}
\hline$A$ & $B$ \\
\hline $\begin{array}{ll}\text { 1. } & \mathrm{M} \\
\text { 2. } & \delta \mathrm{m} \\
\text { 3. } & \{\delta \mathrm{m}\} \mathrm{sk}(\mathrm{A}) \\
\text { 4. } & \{\{\delta \mathrm{m}\} \mathrm{sk}(\mathrm{A}), \mathrm{m}, \\
& \mathrm{A}\} \mathrm{pk}(\mathrm{B}) \\
\text { 5. } & \text { Send }\end{array}$ & 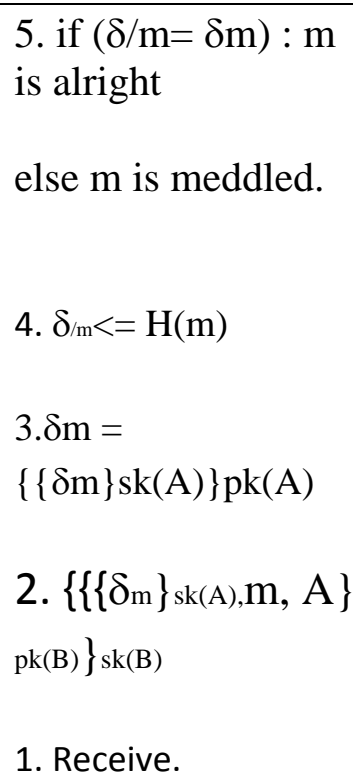 \\
\hline
\end{tabular}

Let us assume,

A : John

B : Carry

$\mathrm{M}$ : message

$\delta \mathrm{m}$ : digest of a message(encrypted)

$\mathrm{H}(\mathrm{m})$ : Hash function of a message

SkA : secret key of A

PkA : public key of A

SkB : secret key of B

$\mathrm{PkB}$ : public key of B

$\delta / \mathrm{m}$ : digest of a message (decrypted).

\subsection{XML Signature}

XML Signature is a protocol explaining the signing of digital information. The XML Signature standard contains protocols for signing sections of XML documents. XML signature probable such possibilities as message reliability. XML Signature produces reliability for data. XML Signature is additionally essential for authentication and non-repudiation. XML Signature is a technology that needs to be implemented appropriately if it is to be an appropriate security application. XML Signature may also be employed for trustworthiness and non-repudiation of WSDL files in order that the definition of Web Service tends to be circulated and subsequently trustworthy. XML Signature provides an alluring means of articulating a Digital Signature through XML data.The structure of an XML signature is:

<signature> <signedInfo>

(canonical form) - to preventing white space.

(signature form)

(<reference(URI=) ? >

(changes) ?

(digest method)

(digest value)
$</$ reference $>)+</$ signedInfo $>$

(signature value)

(keyInfo) ?

(object) *

$</$ signature $>$

Where, ? => 0 or 1 time, $+=>1$ or more time, $*$

$=>0$ or more time which has extracted from the

notion of regular expressions.

\subsection{MPMSD}

Document manufacturing within an workplace is based on a request-reaction-response prototype. A MultiPart MultiSignature Document (MPMSD), DW, produced in a Document formation Workflow (DPW)[26] W, is an n-tuple, $\mathrm{n}=1$, such that $\mathrm{DW}=(\mathrm{d} 1, \mathrm{~d} 2, \mathrm{~d} 3,---, \mathrm{dn})$. Each part di in turn is identified as a 3 -tuple (mi, oi, si), where mi is the comment of the reviewer's $i$, and $\sigma i$ is the signature of si

\subsection{A Scenario}

Within an office system, an employee referred to as originator, creates a document as well as transfers it to some other worker, who analysis, gives remarks as well as forwards it to different employee and so on. This procedure in reply will provide a composite document, comprised of list of comments, supplied by the originator and the evaluators throughout the process. This kind of document is known as Multi-Part Multi-Signature Document (MPMSD). The above mentioned mechanism of MPMSD is shown in the Fig. 3.

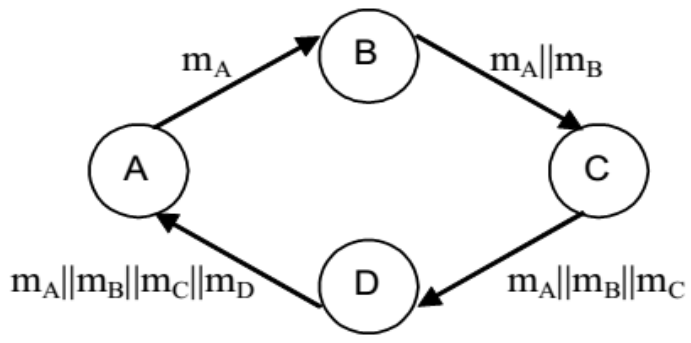

Fig 3 Mechanism of MPMSD

In a manual process, it is similar paper document that is circulated among and the evidence that it has come through appropriate canalize with the addition of variety of reviews accompanied by the signatures of the reviewers.

\section{ASSUMPTIONS AND FUTURE WORK}

In the existing work a review of security concerns in Web service is carried out within a typical security framework. Within this paper, some new security challenges are emphasized. The technology like WS-Routing can be utilized on SOAP messages for protected multi-service hopping. Although analyzing the literature, it is unearthed that data mining techniques[16] can be utilized efficiently for discovering attacks. A new security architecture based upon Web services that assist authentication, authorization and confederation is a central point for future research. Additionally whether XML signature and MPMSD yields security to data and contents are always points for upcoming inquiries. 


\section{REFERENCES}

[1] Cotroneo,D.; Graziano, A; Russo,S.(2004). "Security Requirements in Service Oriented Architectures for Ubiquitous Computing". 2nd Workshop on Middleware for pervasive and Ad-hoc Computing. Toronto. Canada

[2] Gupta, K. N.; Agarwala, K. N. Agarwala, P. A.(2005). Digital Signature: Network Security Practices. PHI Pub. New Delhi

[3] Gutierrez, C.; Medina, E. F.; Piattini, M.(2005). Web Services Enterprise Security Architecture: A Case Study. Fairfax. Virginia. USA

[4] Kahate, A.(2003). Cryptography and Network Security. TATA McGraw Hill

[5] Kearney, P.; Chapman, J.; Edwards, N.; Gifford, M.; He,I. (2004). An Overview of Web Services Security. BT tech. Journal. 22(1): 27-42

[6] Mcintosh, M.; Austel, P.(2005). XML Signature Element Wrapping Attacks and Countermeasures. Fairfax. Virginia. USA

[7] Michael N. H.; Singh, M. P.(2005). Service-Oriented
Computing: Key concepts and principles. IEEE Internet computing. 9(1):75-81

[8] Milanovic, N.; Miroslaw, M. (2004). Current solutionsfor Web-Service Composition. IEEE Internet Computing: 51-59

[9] Neil. M.O. (2003). Web-Service Security. Tata Mcgraw Hill Pub. New York

[10] Available Online: http://www.soapclient.com/ uddiadv.html

[11] 1. Jun-ichi Akahani, Kaoru Hiramatsu, Kiyoshi Kogure, Coordinating Heterogeneous Information Services based on Approximate Ontology Translation, In B. Burg, J. Dale, T. Finin, H. Nakashima,L. Padgham, C. Sierra, and S. Willmott, editors, Agentcities: Challenges in Open Agent Environments, . Springer-Verlag, 2003.

[12] V. Richard Benjamins, Web Services Solve Problems, and Problem-Solving Methods Provide Services, IEEE Intelligent Systems, January/February (2003) 76-77. 